\title{
MONITORING DRIVER DROWSINESS AND STRESS IN A DRIVING SIMULATOR
}

\author{
Maria Rimini-Doering, Dietrich Manstetten, \\ Tobias Altmueller, Ulrich Ladstaetter, Michael Mahler \\ Corporate Research Division \\ Robert Bosch GmbH \\ Stuttgart, Germany \\ E-mail: maria.rimini-doering@de.bosch.com
}

\begin{abstract}
Summary: Driver drowsiness, compounded by the high workloads and stress of the ever-increasing complexity of car and traffic environments, is a major cause of severe accidents. The objective of the project described in this paper is to develop reproducible and flexible methods for studying the relationships between physiological driver states and human-factor issues in a driving environment. For reasons of safety and reproducibility, a laboratory-based driving simulator is being used for the project experiments. Initial experiments were conducted with a cohort of about 60 healthy male subjects aged 22 to 28 under carefully controlled conditions. Performance was measured before, during, and after a $120 \mathrm{~km}$ stretch of stimulus-deprived, foggy highway that was intended to induce fatigue and stress. Across all trials $69 \%$ of the subjects experienced sleep events lasting several seconds, and 7 potentially fatal crashes occurred. Lane tracking behavior degraded by a factor of 2 to 3 prior to each crash. Much of the extensive data acquired by these experiments remains to be analyzed using both standard statistical techniques and highdimensional clustering algorithms. ALISA image-processing software is being applied to video images of the driver eyes and face to detect the onset of sleep and other critical situations.
\end{abstract}

\section{INTRODUCTION}

International statistics identify driver drowsiness as a major cause of severe traffic accidents (Dinges, 2000). In addition, the growing complexity of the car and traffic environment, including the operation of assistance and information systems, can easily exceed driver workload limits and create stress that degrades driving performance (Green, 2001). For this reason, a driver monitoring research project is being conducted at the Corporate Research Division of Robert Bosch GmbH, Germany.

\section{Objective}

The primary objective of this project is to develop reproducible and flexible methods for studying the relationships between physiological driver states and human-factor issues in the design of adaptive driver-assistance and driver-information systems under a variety of driver and driving conditions. This includes the determination of useful sensors and their operating ranges, relevant simulation conditions, and appropriate protocols for conducting rigorously controlled experiments with the human subjects under a variety of driving situations. It is expected that the results and conclusions of these experiments will eventually identify significant driver states and 
their associated physiological signals, leading to the development of non-invasive sensors for reliably monitoring driver states under a variety of conditions.

\section{EXPERIMENT EQUIPMENT}

\section{Driving Simulator}

For reasons of safety and reproducibility, a laboratory-based driving simulator is being used for the project experiments. The hardware and software is based on the commercial simulator STISIM 500W provided by Systems Technology Inc. (Allen, 1998). The driver sits in a fully equipped front half of a Fiat Coupé, controlling the car with a steering wheel with force feedback,

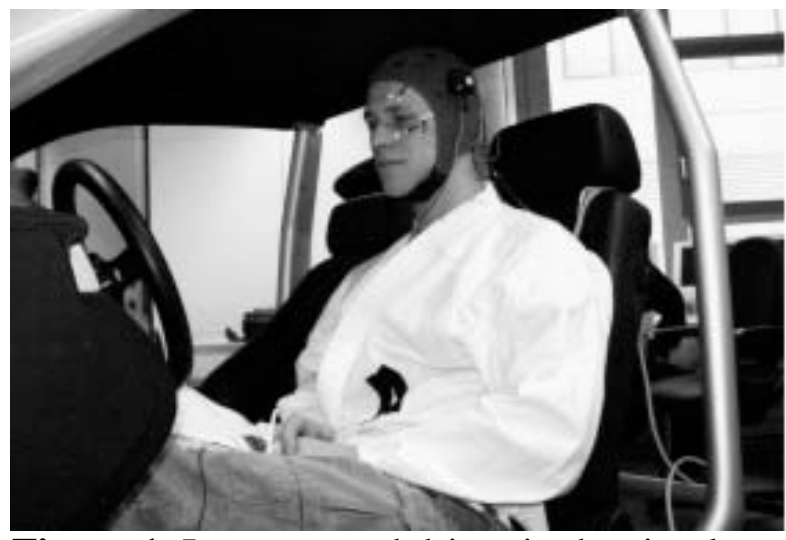

Figure 1. Instrumented driver in the simulator. gear shift, and brake and accelerator pedals. The animated driving scene, which responds dynamically to all driver commands, is projected onto a front display with a $135^{\circ}$ FOV (Figure 1). The simulator generates car noise as a function of engine speed and ground speed. No dynamic force or vibration feedback is given to the driver. The current vehicle conditions (position, speed, pedal and steering wheel actions, etc.) as well as the scenario conditions (highway curvature, fog density , etc.) are recorded every $20 \mathrm{~ms}$ and synchronized with all the outputs of the physiological, video, and environmental sensors.

\section{Sensors and Measurements}

During the experiment trials, several conditions and parameters are recorded for possible correlation with the physical and mental state of the driver, driving behavior, and scenario conditions.

Physiological sensors. A dedicated data-recording system has been developed for this purpose (Pasquarelli, 2001). It can be used both in laboratory-based simulators and in actual mobile (car) environments. Measured parameters include:

a) Electrocardiogram (ECG), three channels;

b) Electroencephalogram (EEG), 13 channels from a standard 10-20 EEG-cap;

c) Electrooculogram (EOG) at one eye, $x$ and $y$ component, one channel each;

d) Skin impedance at the lower arm;

e) Skin temperature;

f) Pulse and oxygen saturation in the blood (transmission sensor on the finger);

g) Respiration frequency with piezoelectric element under the belt;

h) Head movements with a three-axis accelerometer.

Quickly changing parameters a) through d) are sampled every millisecond; all others are sampled every 100 milliseconds.

Movement sensors. A grayscale video camera records the driver's face to monitor eye and eyelid movements at 25 frames per second and a resolution of 128x128 pixels. Its sensitivity in the near 
infrared (using infrared photodiodes) allows meaningful recording under reduced lighting conditions (night, fog, etc.). Two other ordinary video cameras record head movements of the subject and a lateral view of the torso and head of the subject to monitor stretching, yawning, etc.

Environment sensors. Laboratory environment conditions are sampled every 100 milliseconds, including room temperature, air humidity, $\mathrm{CO}_{2}$ and $\mathrm{O}_{2}$ concentrations in the air, etc.

\section{EXPERIMENTS}

Special care was taken throughout the design and conduct of the first set of experiments with human subjects to adhere strictly to the Scientific Method, to enforce a consistent research methodology, and to maintain rigorous experiment controls to minimize error and bias (Bock, 2002).

\section{Block Design}

Treatment Design. Driver stress occurs when a period of unchanging driving conditions and little sensory stimulation (which causes drowsiness) is interrupted by sudden sensory overload. For this reason three contrasting treatments (simulator scenarios) were devised:

baseline: a simple $4 \mathrm{~km}$ segment with no fog, no curves, and no traffic

stress: a $120 \mathrm{~km}$ segment in fog (50m visibility) interrupted occasionally with gentle curves and slopes with little or no traffic

control and test: a $10 \mathrm{~km}$ segment with sudden, large changes in curvature and slope in a lively street environment (oncoming traffic, pedestrians, etc.), partially with fog.

Trial Design. The driving course for each experiment trial included the above treatments in the following sequence: baseline, control, stress, test, baseline. Thus, each trial had a total driving distance of $148 \mathrm{~km}$. The stress region was actually comprised of three identical sections (A, B, C) repeated four times (A, B, C, A, B, C, A, B, C, A, B, C). This will allow driver behavior to be evaluated at four different times during the stress treatment under identical highway conditions to observe progressive degradation of driving performance.

\section{Cohort Selection}

A cohort of about 60 healthy men (students) between 22 and 28 years old was selected from 220 applicants using a simulator-sickness test and five questionnaires: medical status, psychological status, driving and sleeping habits, emotional assessment, and subjective reactions to the simulator. A short interview with a medical doctor to cull problematic subjects completed the selection process.

Each subject was encouraged to overlook any lack of realism of the simulation and to drive according to German road traffic regulations (speed limits, etc.). The subjects were not told about the length or distance of the trial in advance. The objectives and goals of the experiment (drowsiness recognition, etc.) were not mentioned and questions about these topics were politely discouraged. 


\section{Experiment Trials}

All trials were conducted between November 2000 and June 2001. All subjects were prepared and all trials were controlled and monitored by the same supervisor (a trained nurse), who remained in an adjoining room during the trial to avoid distracting or cueing driver. All of the interaction with each subject was strictly governed by a detailed protocol with 180 individual steps (Ladstaetter, 2001).

Each subject spent about seven hours at the Bosch Driving Simulator facility. When he arrived at the laboratory at noon, the subject was given a short introduction to the agenda for the afternoon, followed by a full meal with no alcoholic drinks or coffee. After returning to the laboratory, the installation of the physiological electrodes and sensors lasted about an hour. The subject was then asked to drive the simulator for a few minutes to familiarize himself with its operation. The formal driving trial required approximately three hours (148 km at approximately $50 \mathrm{~km} / \mathrm{h}$ ), which was intentionally conducted in the early afternoon to coincide with the second performance minimum in the day. A short debrief session completed the experiment. INITIAL RESULTS AND OBSERVATIONS

\section{Sleep and Accidents}

Because the acquisition of information about the physiological states of drowsy drivers was an essential requirement for this experiment, the fact that a high percentage of subjects exhibited one or more sleep episodes served us as a positive confirmation of a sound experiment design.

Sleep episodes. Sleep episodes were defined as situations in which the driver keeps his eyes closed for longer than one second. At a speed of about $50 \mathrm{~km} / \mathrm{h}$, the car travels a distance of about 15 meters during one second. Several hundred such episodes were recorded among 69\% of the subjects, the longest being around 8 seconds, corresponding to a distance of about 120 meters (Hargutt, 2000). Figure 2 shows the distribution of these sleep episodes over the distances covered. The number of sleep episodes increases with the time spent driving in the foggy, stimulusdeprived Stress region, reaching a maximum between 60 and $110 \mathrm{~km}$ (1 or 2 hours). Several explanations for this maximum are possible:

1) Nine subjects fell asleep and caused severe accidents. Such subjects were not permitted to complete the trial and thus provided no further contribution to the overall statistics.

2) Some drivers fell asleep and came very close to causing an accident, but managed to keep the car under control and continue on with the trial with sharpened awareness.

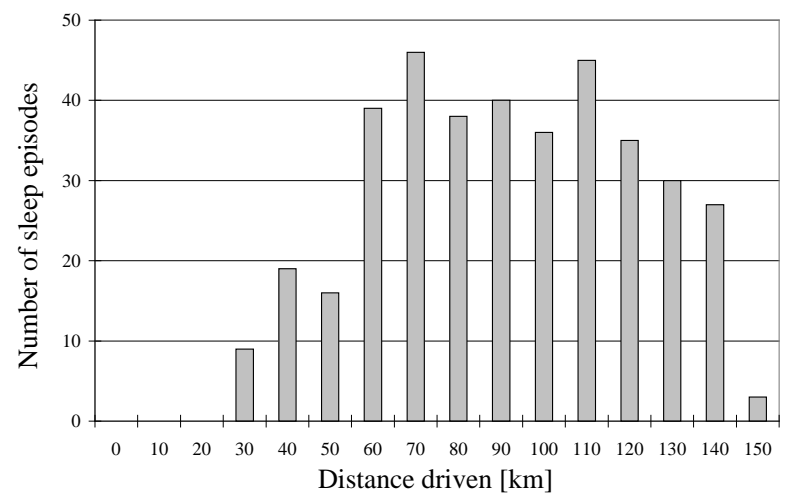

Figure 2. Sleep episodes during simulation.

3) The busy Test region at $130 \mathrm{~km}$ recaptures the driver's attention and rejuvenates him somewhat.

Accidents Caused by Sleep. Four of the nine severe accidents were the direct and sole result of the driver falling asleep. Each drove with closed eyes for several seconds and either crashed into oncoming traffic or left the pavement without steering or braking to avoid it.

Accidents Caused by Drowsiness. Three of the nine severe accidents occur in the Test region and were probably caused by degraded judgement or reaction time, such as not braking properly or 
quickly enough and hitting a crossing pedestrian. The same driving task during the Control region caused no problems in any trials. Thus, it may be tentatively concluded that the accident resulted from the drowsiness and stress induced during the Stress region.

\section{Eye and Eyelid Movement}

To automatically detect eyelid closure, a proprietary software package ALISA (Adaptive Learning Image and Signal Analysis) has been applied to the IR camera images (Bock, 1998). A sample of the initial results is shown in Figure 3. Four sequential images of a driver's face are shown next to their corresponding ALISA normality maps. The size of the

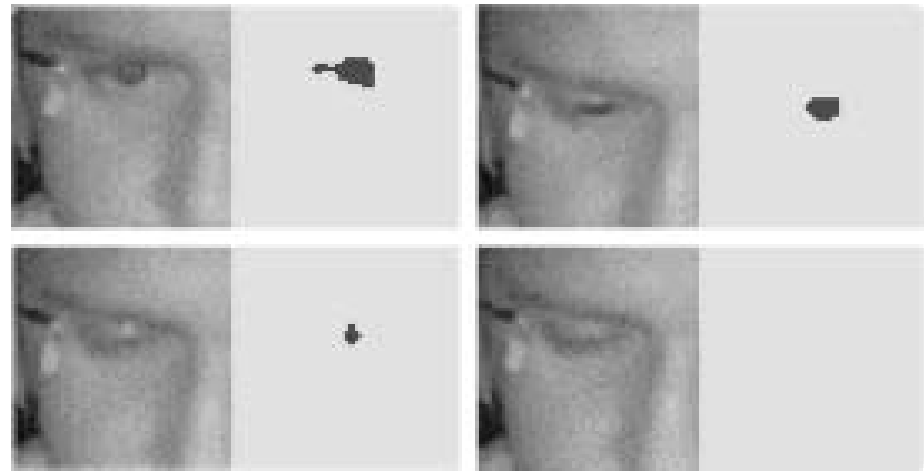

Figure 3. ALISA analysis of an eye-closing sequence. dark spot varies directly with the size of the exposed area of the eye; the spot disappears when the eyelid closes completely. A duration threshold can differentiate normal blinks from sleep onset.

\section{Lane Tracking Behavior}

An initial analysis of the lane-tracking sensor data also reveals some possible indicators of stress. As Figure 4 shows, the standard deviation of the lateral offset of the car from the center line is small during the nonstressful Baseline (first $4 \mathrm{~km}$ ), but more than doubles its value in the challenging Control region as far as $\mathrm{km} \mathrm{14}$. It then falls abruptly as the Stress region begins and the driver reduces speed considerably. Thereafter, the value starts slowly increasing again, possibly as drowsiness and boredom set in. After a "near accident" at about $90 \mathrm{~km}$, it decreases as the driver "wakes up", but soon starts increasing again, spiking during the final Test region, starting at $134 \mathrm{~km}$.

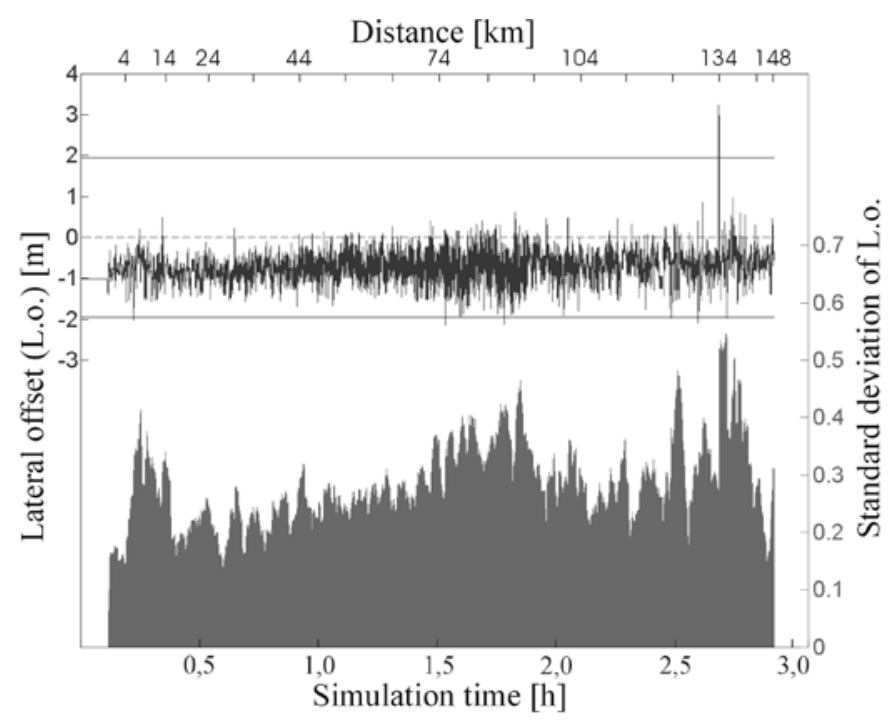

Figure 4. Lane position deviation (top) and its standard deviation over the last 2 minutes (bottom) as a function of elapsed simulation time and distance.

\section{CONCLUSIONS}

Several different initial perspectives of the recorded data yields a coherent picture of significant events during the trials. In particular, the high number of sleep and drowsiness episodes confirms that the experiment design does, in fact, cause sufficient drowsiness and stress to be able to measure its effects. The degradation of the lane tracking capability shows promise for assessing the state of the driver. The ALISA normality maps of the IR images of the driver's face appear to provide a reliable indicator for closed-eye states. Correlation with other physiological parameters are expected from the high-dimensional clustering analysis in late summer 2001. It is planned to repeat part of the experiment with a double instrumented vehicle on a Bosch-owned test track as 
well as in the driving simulator laboratory with cohorts of different ages, sexes, etc.

\section{ACKNOWLEDGEMENTS}

We gratefully acknowledge the contributions of P. Bock, Professor of Machine Intelligence and Cognition (Department of Computer Science at The George Washington University, Wash. DC) to the experiment design. We also thank Drs. S.N. Erné and A. Pasquarelli (Institute for Biomedical Technology in Ulm, Germany) for the development of the physiological measuring equipment and Prof. F. Lang (Department of Physiology, University of Tübingen, Germany) for his medical advice.

\section{REFERENCES}

[1] R.W. Allen, T. Rosenthal, B.L. Aponso, D.H. Klyde, F.G. Anderson, J.P. Chrstos, "A low Cost PC Based Driving Simulator for Prototyping and Hardware-in-the-Loop Applications", SAE Paper No. 98-0222, Spec. Pub. 1361, 1998.

[2] P. Bock, Getting it Right: R\&D Methods for Science and Engineering, Academic Press, 2002.

[3] P. Bock, "ALISA: Adaptive Learning Image and Signal Analysis", Proceedings of the SPIE Applied Imagery Pattern Recognition Conference, Washington DC, October 1998.

[4] D. Dinges, "Accidents and Fatigue", Proc. of the Internat. Conference The Sleepy Driver and Pilot, Stockholm, 2000

[5] P. Green, "International Incidents", ITS World, Jan./Feb. 2001

[6] V. Hargutt, H.-P. Krüger, "Compensation for Drowsiness and Fatigue. A Driving Simulation Study", Proceedings of the International Conference of Traffic and Transportation Psychology (ICTTP), Bern, Switzerland, 2000.

[7] U. Ladstaetter, Pilotstudie zur Fahrerzustandserkennung im Kraftfahrzeug, Masters Thesis, Stuttgart, Germany,2001.

[8] A. Pasquarelli, M. Rimini-Doering, S.N. Erné, “Datenerfassungssystem für die Aufnahme relevanter physiologischer Parameter im Fahrsimulator", Proceedings of 35th Conference of the German Biomedical Society (DGBMT), 2001. 